\title{
Utilización de nuevas herramientas para la evaluación de la asignatura Estadística Empresarial I: matrices de especificidades y hojas de evaluación.
}

\author{
Antonio Miguel Márquez Durán(1) \\ (1) Departamento de Economía, Métodos Cuantitativos e $H^{a}$ Económica, \\ Universidad Pablo de Olavide, Ctra. de Utrera, 1, 41013 Sevilla, \\ Presentado en eXIDO19 (2019)
}

\section{RESUMEN}

En este trabajo queremos mostrar una serie de herramientas que nos sirvan para comprobar la adquisición, por parte del alumnado de Estadística Empresarial I, de ciertas competencias, de una forma sencilla pero a la vez rigurosa.

Palabras clave: adquisición y evaluación de competencias, matrices de especificidades, hojas de evaluación.

\section{INTRODUCCIÓN}

Como es bien conocido, el nuevo modelo de formación implantado en las universidades españolas está centrado en el trabajo del estudiante y en la adquisición de competencias. Determinar dichas competencias dentro de un determinado perfil no ha resultado tan difícil (las competencias aparecen recogidas en las guías docentes de las asignaturas, tanto a nivel general como al específico de la misma), lo que sí lo está siendo, en la mayoría de las ocasiones, es evaluar alguna de ellas. Nuestro objetivo es introducir nuevas herramientas para llevar a cabo esta evaluación.

\section{METODOLOGÍA}

El planteamiento de una evaluación exige una reflexión previa sobre un conjunto de criterios que son los que condicionan su eficacia. No se puede improvisar, sino que debe ser fruto de un trabajo organizado, riguroso y responsable. Para llevar a cabo esta evaluación de manera que podamos cumplir con unos mínimos de validez y calidad, vamos a diseñar matrices de especificidades, indicando las unidades de competencias que vamos a evaluar, el peso ponderativo de los conocimientos teóricos y prácticos, etc. 
Además, para las prácticas de informática utilizaremos las hojas de evaluación, pretendiendo evaluar habilidades y destrezas adquiridas por el alumno para desarrollar una determinada tarea. Con las hojas de evaluación calificamos ponderando la importancia de una serie de operaciones que se presentan en forma dicotómica (lista de cotejo) y/o en forma de grados (escalas). El diseño de una Hoja de Evaluación ha de ser cuidadoso ya que es necesario que esté bien realizada para conseguir el máximo de objetividad. Supone analizar detenidamente y seleccionar todos aquellos aspectos que el formador 0 evaluador tiene que observar para certificar al alumno. No podemos olvidar que dicho diseño parte de la Unidad de Competencias y del peso ponderativo que le hemos otorgado en la Matriz de Especificidades.

\section{RESULTADOS}

En la asignatura Estadística Empresarial I del Grado de Administración y Dirección de Empresas, se evalúan las Enseñanzas Básicas (EB) y las Enseñanzas Prácticas y de Desarrollo (EPD) impartidas durante el curso, asignándole los siguientes pesos:

- Enseñanzas Básicas (50\%). Hay una prueba escrita al final del semestre, que consta de preguntas teóricas (10\%), y otra parte práctica (40\%). La parte teórica está formada por un test de 10 preguntas con 4 posibles respuestas y con la característica de que los fallos están penalizados con una puntuación de $1 / 4$ de una respuesta acertada. La parte práctica consta de 4 problemas a resolver.

- Enseñanzas Prácticas y de Desarrollo (50\%). Esta parte sigue el procedimiento de evaluación continua. Está formada por trabajos individuales y en grupo que tienen que entregar los alumnos $(30 \%)$ y por la realización de tres prácticas de informática evaluadas con una valoración del $20 \%$. Los trabajos individuales consisten en la evaluación de los contenidos de la asignatura mediante la realización de 4 pruebas tipo test con 5 preguntas con 3 respuestas alternativas, de la que penalizan las respuestas mal contestadas con $1 / 3$ de cada respuesta acertada. La valoración de estos 4 test es de un $20 \%$ de la nota total. El trabajo en grupo consiste en la exposición de un tema relacionado con la estadística. Se valora con un $10 \%$ de la nota total.

La evaluación de las enseñanzas básicas y de las actividades prácticas y de desarrollo ha servido principalmente para hacer un seguimiento de los conocimientos adquiridos por los alumnos. En la exposición oral de los trabajos realizados por los estudiantes, se ha medido la adquisición de otras competencias más difíciles de contrastar en un examen escrito: además de conocer el contenido del trabajo, se ha comprobado la capacidad de síntesis del alumno, su habilidad para contestar preguntas relacionadas con el tema expuesto, su capacidad de trabajo en grupo, su capacidad de exposición pública, etc. Se ha tratado de desarrollar una serie de competencias generales 
tanto de tipo instrumental, interpersonal como sistémicas. Entre las de tipo instrumental destacamos el análisis y síntesis, organización y planificación, comunicación oral y escrita, resolución de problemas y gestión de la información. En las de tipo interpersonal la capacidad crítica y autocrítica y la habilidad para trabajar de forma autónoma. Y por último, en las de tipo sistémico enfatizamos cómo aplicar los conocimientos a la práctica, adaptación a nuevas situaciones y capacidad de aprender, entre otras.

Para llevar a cabo esta evaluación de manera que pudiésemos cumplir con unos mínimos de validez y calidad, hemos empezado por el diseño de una Matriz de Especificaciones.

\begin{tabular}{|c|c|c|c|c|c|c|c|}
\hline & & \multirow[b]{3}{*}{$\begin{array}{l}\text { Pesos } \\
\text { Pondera } \\
\text { tivos }\end{array}$} & \multirow{2}{*}{\multicolumn{4}{|c|}{$\begin{array}{l}\text { Prueba de conocimientos } \\
\text { (No ítems total y por niveles } \\
\text { taxonómicos) }\end{array}$}} & \\
\hline & \multirow[b]{2}{*}{$\begin{array}{c}\text { Unidades } \\
\text { de } \\
\text { competen } \\
\text { cia }\end{array}$} & & & & & & \multirow{2}{*}{$\begin{array}{c}\begin{array}{c}\text { Prueba } \\
\text { Práctica }\end{array} \\
\begin{array}{c}\text { Identific } \\
\text { ación de } \\
\text { las } \\
\text { práctica } \\
\text { s }\end{array} \\
\end{array}$} \\
\hline & & & $\begin{array}{l}\mathrm{N}^{\circ} \\
\text { Ite } \\
\text { ms }\end{array}$ & $\begin{array}{l}\text { Conocimi } \\
\text { entos }\left(\mathrm{N}^{\circ}\right. \\
\text { Items) }\end{array}$ & $\begin{array}{l}\text { Compre } \\
\text { nsión } \\
\text { (No }^{\circ} \\
\text { Items) }\end{array}$ & $\begin{array}{c}\text { Aplica } \\
\text { ción } \\
\left(N^{\circ}\right. \\
\text { Items })\end{array}$ & \\
\hline \multirow{2}{*}{ 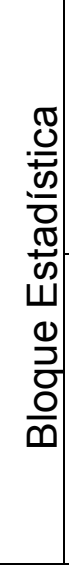 } & $\begin{array}{l}\text { 1.Distribuci } \\
\text { ones de } \\
\text { frecuencias } \\
\text { unidimensi } \\
\text { onales }\end{array}$ & \multirow[b]{2}{*}{$32.5 \%$} & \multirow[b]{2}{*}{13} & \multirow[b]{2}{*}{4} & \multirow[b]{2}{*}{3} & \multirow[b]{2}{*}{6} & \multirow{2}{*}{$\begin{array}{c}\text { Práctica } \\
\text { de } \\
\text { informáti } \\
\text { ca } 1 \\
\text { Trabajo } \\
\text { final }\end{array}$} \\
\hline & $\begin{array}{l}\text { 2.Distribuci } \\
\text { ones de } \\
\text { frecuencias } \\
\text { bidimensio } \\
\text { nales. } \\
\text { Regresión } \\
\end{array}$ & & & & & & \\
\hline \multirow{2}{*}{\multicolumn{2}{|c|}{$\begin{array}{l}\text { 3.Números } \\
\text { índices y } \\
\text { Series } \\
\text { temporales }\end{array}$}} & \multirow[t]{2}{*}{$22.5 \%$} & \multirow[t]{2}{*}{9} & \multirow[t]{2}{*}{4} & \multirow[t]{2}{*}{1} & \multirow[t]{2}{*}{4} & $\begin{array}{c}\text { Práctica } \\
\text { de } \\
\text { informáti } \\
\text { ca 2 }\end{array}$ \\
\hline & & & & & & & $\begin{array}{c}\text { Trabajo } \\
\text { final }\end{array}$ \\
\hline & $\begin{array}{l}\text { 4.Fenómen } \\
\text { os } \\
\text { Aleatorios. } \\
\text { Probabilida } \\
\text { d }\end{array}$ & $22.5 \%$ & 9 & 2 & 3 & 4 & \multirow{2}{*}{$\begin{array}{c}\text { Práctica } \\
\text { de } \\
\text { informáti } \\
\text { ca } 3\end{array}$} \\
\hline & $\begin{array}{l}\text { 5.Variables } \\
\text { Aleatorias. }\end{array}$ & $22.5 \%$ & 9 & 2 & 3 & 4 & \\
\hline
\end{tabular}




\begin{tabular}{|c|c|c|c|c|c|c|}
\hline $\begin{array}{l}\text { Modelos } \\
\text { probabilísti } \\
\text { cos }\end{array}$ & & & & & & \\
\hline Totales & $100 \%$ & 40 & 12 & 10 & 18 & \\
\hline
\end{tabular}

De todas formas, de entre todas las estrategias utilizadas para que el alumno pueda alcanzar los objetivos de la asignatura me gustaría destacar la correspondiente a la evaluación continua dentro de las Enseñanzas Prácticas y de Desarrollo, y dentro de ésta el trabajo final en grupo, única tarea del curso que no es de carácter individual, y que por tanto, va a permitir adquirir competencias que de otra forma, sería muy difícil hacerlo. A continuación, vamos a describir de forma más explícita en qué ha consistido esta tarea y adjuntamos la Hoja de Evaluación que nos ha permitido constatar el grado de consecución de los objetivos de la misma.

El trabajo ha consistido en aplicar los conocimientos adquiridos en los temas 1, 2 y 3 sobre una base de datos real que el grupo debe seleccionar en los siguientes enlaces:

- Instituto Nacional de Estadística (INE), http://www.ine.es.

- Instituto de Estadística y Cartografía de Andalucía,

https://www.juntadeandalucia.es/institutodeestadisticaycartografia/temas/index$\underline{\text { est.htm }}$

- Eurostat, https://ec.europa.eu/eurostat/home?

Los grupos han estado compuestos por 4 personas. Ha habido que seleccionar una base de datos temporal con varias (al menos dos) variables económicoempresariales. A dichos datos, ha habido que realizarle un análisis descriptivo, de regresión lineal simple o múltiple y un análisis temporal comparativo. Se ha debido realizar predicciones a corto plazo, proporcionando la fiabilidad de éstas. Por otro lado, se ha debido calcular indicadores y/o tasas de variación para las variables más relevantes. Para ello, se ha utilizado el software utilizado en las prácticas informáticas (SPSS/PASW y MS Excel).

La extensión del análisis no debe superar las 20 páginas escritas (portada, índice, gráficos y tablas incluidos) ni el fichero debe tener un tamaño superior a los $2 \mathrm{Mb}$. El formato debe ajustarse a la plantilla que se adjunta (Analisis_Template.dot).

Además el trabajo tendrá que presentarse ante los compañeros en la última clase de EB del curso con transparencias (fichero de PowerPoint o similar).

Se ha valorado de la siguiente forma:

- Corrección técnica (50\%): coherencia, propiedad en el uso del lenguaje técnico y utilidad de las conclusiones.

- Formato (25\%): Adecuación de la presentación a las normas establecidas. 
- Presentación en clase (25\%): Durante un máximo de 10 minutos, cada grupo expondrán en clase con ayuda de transparencias (fichero de PowerPoint o similar) el trabajo realizado debiendo responder a las preguntas que se le realicen relativas al trabajo y la exposición. Se valorarán especialmente las conclusiones y sus justificaciones, no así los detalles relativos al manejo de SPSS y MS Excel. Cualquier miembro del grupo puede ser seleccionado por el profesor para exponer el trabajo.

Los trabajos se valorarán globalmente con 1 punto y la nota será común para todo el grupo.

La siguiente tabla corresponde a la Hoja de Evaluación de esta actividad:

\begin{tabular}{|c|c|c|c|c|}
\hline $\begin{array}{c}\text { Activid } \\
\text { ades } \\
\text { (Variabl } \\
\text { es a } \\
\text { valorar) }\end{array}$ & Factores & Cotejos y escalas & $\begin{array}{c}\text { Pun } \\
\text { t. } \\
\text { Máx } \\
\text {. }\end{array}$ & $\begin{array}{l}\text { Punt. } \\
\text { Obteni } \\
\text { da }\end{array}$ \\
\hline \multirow[t]{2}{*}{$\begin{array}{l}\text { Correcc } \\
\text { ión } \\
\text { Técnica } \\
\text { (50\% } \\
\text { del } \\
\text { total) }\end{array}$} & $\begin{array}{l}\text { Coherencia } \\
\text { con lo que se } \\
\text { pide }\end{array}$ & 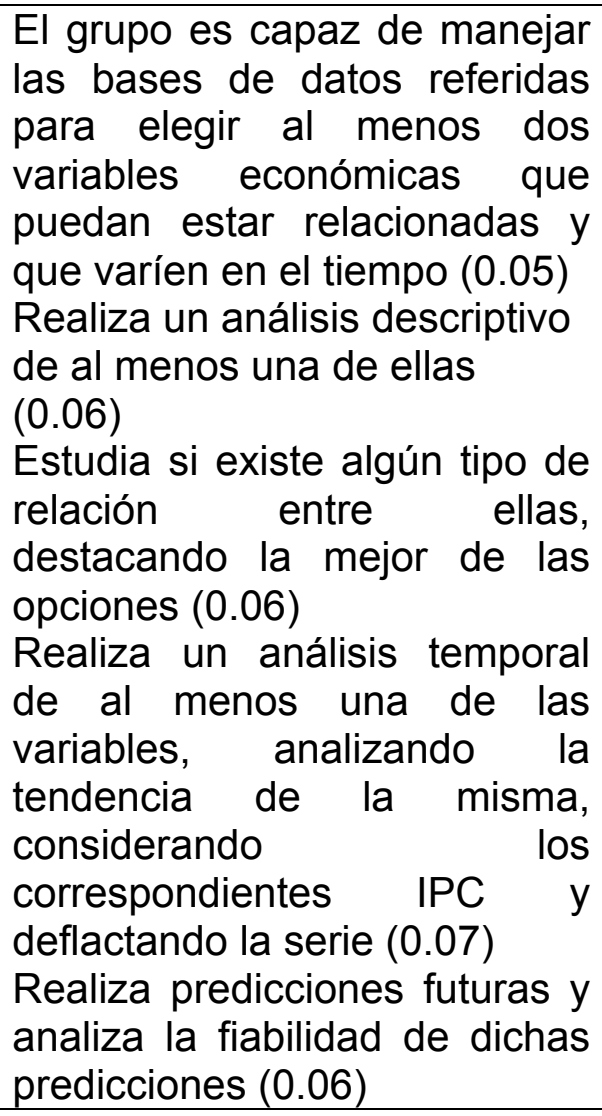 & 0.3 & \\
\hline & $\begin{array}{l}\text { Propiedad en } \\
\text { el uso del } \\
\text { lenguaje }\end{array}$ & $\begin{array}{l}\text { El grupo conoce los nombres } \\
\text { de los principales parámetros } \\
\text { estadísticos estudiados }(0.04)\end{array}$ & 0.1 & \\
\hline & $\begin{array}{l}\text { técnico y de } \\
\text { las } \\
\text { herramientas }\end{array}$ & \multicolumn{3}{|l|}{$\begin{array}{l}\text { Representa la información en } \\
\text { forma de tablas o gráficos } \\
\text { cuando la ocasión lo requiere }\end{array}$} \\
\hline
\end{tabular}




\begin{tabular}{|c|c|c|c|}
\hline & informáticas & $\begin{array}{l}(0.03) \\
\text { Utiliza los programas } \\
\text { informáticos estudiados para } \\
\text { obtener resultados }(0.03)\end{array}$ & \\
\hline & Conclusiones & $\begin{array}{l}\text { El grupo no se queda en el } \\
\text { mero cálculo de los diferentes } \\
\text { parámetros estadísticos sino } \\
\text { que los interpreta }(0.1)\end{array}$ & 0.1 \\
\hline $\begin{array}{c}\text { Format } \\
\text { o } \\
(25 \% \\
\text { del } \\
\text { total) }\end{array}$ & $\begin{array}{l}\text { Adecuación de } \\
\text { la } \\
\text { presentación a } \\
\text { las normas } \\
\text { establecidas. }\end{array}$ & $\begin{array}{l}\text { El trabajo se ajusta a la } \\
\text { plantilla y cumple las normas } \\
\text { de extensión y tamaño }(0.15) \\
\text { La redacción mantiene un } \\
\text { buen léxico, no se detectan } \\
\text { faltas de ortografía ni de } \\
\text { sintaxis }(0.1)\end{array}$ & 0.25 \\
\hline $\begin{array}{l}\text { Present } \\
\text { ación } \\
\text { en } \\
\text { clase } \\
(25 \% \\
\text { del } \\
\text { total })\end{array}$ & $\begin{array}{l}\text { Exposición } \\
\text { adecuada del } \\
\text { trabajo }\end{array}$ & 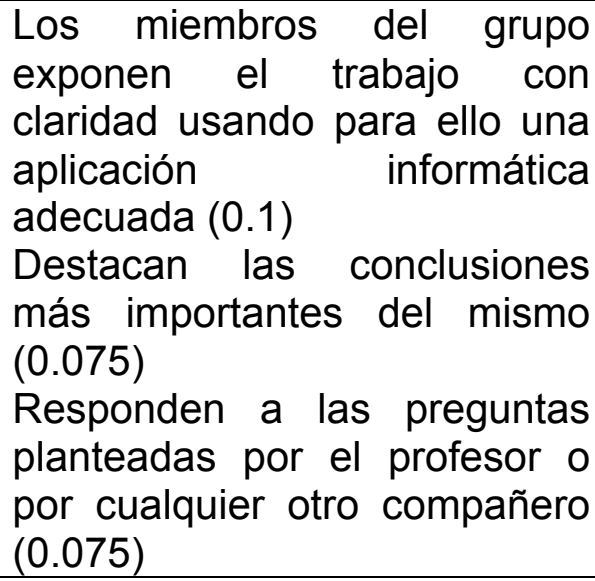 & 0.25 \\
\hline \multicolumn{3}{|r|}{ Total: } & 1 \\
\hline \multicolumn{2}{|c|}{ Factores correctores: } & $\begin{array}{llr}\text { Todos los } & \text { alumnos } \\
\text { demuestran haber } & \text { trabajado } \\
\text { en el seno del grupo } & \\
\end{array}$ & $\begin{array}{l}+/- \\
0.1\end{array}$ \\
\hline \multicolumn{4}{|c|}{ Total (máximo 1punto): } \\
\hline
\end{tabular}

La utilización de estas herramientas nos ha permitido realizar la evaluación de forma más sencilla pero a la vez rigurosa de la correspondiente actividad. Nuestra intención para los próximos cursos es desarrollarlas para el resto de actividades programadas en la asignatura.

\section{CONCLUSIONES}

Estas nuevas herramientas nos han permitido comprobar de una manera más fácil pero a la vez rigurosa si el alumno está alcanzando las competencias correspondientes.

\section{REFERENCIAS}


Innovaciones de los sistemas de evaluación

- Rodríguez Gómez, G. e Ibarra Sáiz, M. S. (Ed.). (2011). e-Evaluación orientada al e-Aprendizaje estratégico en Educación Superior. Madrid: Narcea.

- RubiStar: http://rubistar.4teachers.org/

- Servicio web para la e-Evaluación integrable en Sistemas de Gestión de Aprendizaje (LMS): http://evalcomix.uca.es/ 